\title{
Chapter 7 \\ Difference and Sensitivity Analyses of the LEAP-2017 Experiments
}

\author{
Nithyagopal Goswami, Mourad Zeghal, Bruce L. Kutter, Majid T. Manzari, \\ Tarek Abdoun, Trevor Carey, Yun-Min Chen, Sandra Escoffier, \\ Stuart K. Haigh, Wen-Yi Hung, Dong-Soo Kim, Seong-Nam Kim, \\ Evangelia Korre, Ting-Wei Liao, Kai Liu, Gopal S. P. Madabhushi, \\ Srikanth S. C. Madabhushi, Mitsu Okamura, Asri Nurani Sjafruddin, \\ Tetsuo Tobita, Kyohei Ueda, Ruben Vargas, and Yan-Guo Zhou
}

\author{
N. Goswami · M. Zeghal $(\bowtie) \cdot$ T. Abdoun · E. Korre \\ Department of Civil and Environmental Engineering, Rensselaer Polytechnic Institute, Troy, NY, USA \\ e-mail: zeghal@rpi.edu \\ B. L. Kutter · T. Carey \\ Department of Civil and Environmental Engineering, University of California, Davis, CA, USA \\ M. T. Manzari \\ Department of Civil and Environmental Engineering, George Washington University, \\ Washington, DC, USA \\ Y.-M. Chen · Y.-G. Zhou \\ Department of Civil Engineering, Zhejiang University, Hangzhou, China \\ S. Escoffier \\ IFSTTAR, GERS, SV, Bouguenais, France
}

S. K. Haigh · G. S. P. Madabhushi · S. S. C. Madabhushi

Department of Engineering, Cambridge University, Cambridge, UK

W.-Y. Hung · T.-W. Liao

Department of Civil Engineering, National Central University, Taoyuan, Taiwan

D.-S. Kim

Department of Civil and Environmental Engineering, Korea Advanced Institute of Science and Technology, Daejeon, South Korea

S.-N. Kim

Water Management Department, Korea Advanced Institute of Science and Technology,

Daejeon, South Korea

K. Liu

Institute of Geotechnical Engineering, Zhejiang University, Hangzhou, China

M. Okamura · A. N. Sjafruddin

Department of Civil Engineering, Ehime University, Matsuyama, Japan

T. Tobita

Department of Civil Engineering, Kansai University, Osaka, Japan

K. Ueda $\cdot$ R. Vargas

Disaster Prevention Research Institute, Kyoto University, Kyoto, Japan

(C) The Author(s) 2020

B. Kutter et al. (eds.), Model Tests and Numerical Simulations of Liquefaction

and Lateral Spreading, https://doi.org/10.1007/978-3-030-22818-7_7 


\begin{abstract}
The experimental results of LEAP (Liquefaction Experiments and Analysis Projects) centrifuge test replicas of a saturated sloping deposit are used to assess the sensitivity of soil accelerations to variability in input motion and soil deposition. A difference metric is used to quantify the dissimilarities between recorded acceleration time histories. This metric is uniquely decomposed in terms of four difference component measures associated with phase, frequency shift, amplitude at $1 \mathrm{~Hz}$, and amplitude of frequency components higher than $2 \mathrm{~Hz}(2+\mathrm{Hz})$. The sensitivity of the deposit response accelerations to differences in input motion amplitude at $1 \mathrm{~Hz}$ and $2+\mathrm{Hz}$ and cone penetration resistance (used as a measure reflecting soil deposition and initial grain packing condition) was obtained using a Gaussian process-based kriging. These accelerations were found to be more sensitive to variations in cone penetration resistance values than to the amplitude of the input motion $1 \mathrm{~Hz}$ and $2+\mathrm{Hz}$ (frequency) components. The sensitivity functions associated with this resistance parameter were found to be substantially nonlinear.
\end{abstract}

\title{
7.1 Introduction
}

The Liquefaction Experiments and Analysis Projects (LEAP) are an ongoing series of international collaborations to produce high-quality (centrifuge) experimental data of saturated soil systems and to use this data to validate and assess the performance of constitutive models and numerical tools used in soil liquefaction analyses (Manzari et al. 2018). In 2017, the LEAP exercise involved repeating the same centrifuge test of a sloping deposit at nine centrifuge facilities in China (Zhejiang University, ZJU), France (Institut Français des Sciences et Technologies des Transports, de l'Aménagement et des Réseaux, IFSTTAR), Japan (Ehime University and Kyoto University, KyU), Korea (KAIST University), Taiwan (National Taiwan University, NCU), the UK (Cambridge University, CU), and the USA (UC Davis, UCD, and Rensselaer, RPI). In addition to the main goal of numerical model validation, the tests are aimed at assessing the repeatability, reproducibility, and sensitivity of experimental results among the different facilities.

Assessing the repeatability and reproducibility of the conducted centrifuge experiments requires metrics to quantify the similarities and differences among both the recorded input motions and the responses of the test replicas. A sensitivity analysis may then be used to evaluate how the different input motions and other uncertainties affect the observed soil response. This article proposes a new approach to identify and quantify the differences between time histories of input or response quantities, such as accelerations, velocities, and displacements, provided by experiments and centrifuge tests. The differences are decomposed in terms of measures associated with phase, frequency shift, amplitude at $1 \mathrm{~Hz}$, and amplitude of frequency components higher than $2 \mathrm{~Hz}(2+\mathrm{Hz})$. This approach is used herein to assess the differences and similarities between input accelerations achieved during 26 centrifuge test replicas of the same sloping deposit and evaluate the sensitivity of the experimental results to differences in input motion and deposition. 


\subsection{Experiment Overview}

The LEAP-2017 centrifuge model is a deposit of Ottawa F-65 sand sloping at an angle of $5^{\circ}$ to the horizontal and having a height of $4 \mathrm{~m}$ at mid-slope (Fig. 7.1). The sand was deposited through pluviation in a level rigid container to achieve a range of mass densities (with a reference mean value of $1652 \mathrm{~kg} / \mathrm{m}^{3}$ ). The corresponding relative densities varied from about 50 to $85 \%$ (evaluated using the minimal and maximal densities reported by Kutter et al. (2018, 2019), as displayed in Fig. 7.2).

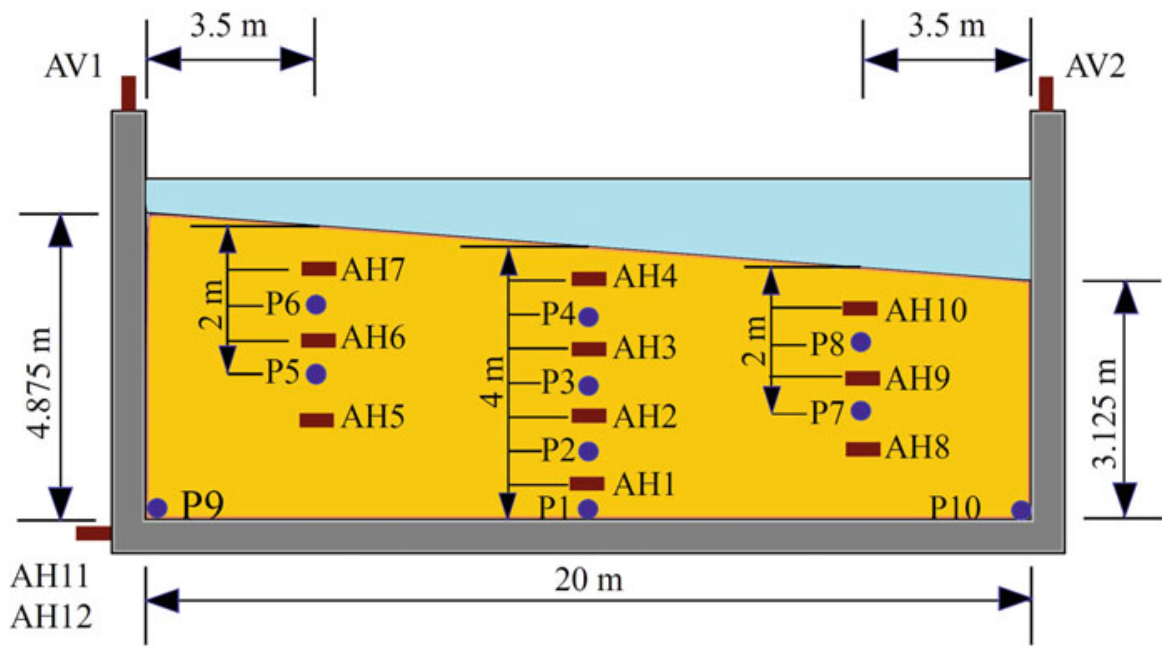

- Accelerometer (AH)

- Pore Pressure Transducer (P)

Fig. 7.1 Schematic of the LEAP-2017 centrifuge model (dimensions are in prototype units)



Fig. 7.2 Relative density of the LEAP-2017 tests conducted at the nine centrifuge facilities 
The deposits were saturated with a viscous fluid to achieve the same prototype hydraulic conductivity at the nine facilities. The models were instrumented with an extensive array of accelerometers (AH1-AH12, AV1, and AV2, with AH11 and AH12 measuring the horizontal input motion at the base of the model) and pore pressure transducers (P1 to P10), as shown in Fig. 7.1. Surface markers were used to measure the permanent lateral displacements (by surveying the location of the markers before and after shaking, as described in Kutter et al. 2018). A CPT (cone penetration test) was used during most of the centrifuge tests to characterize the deposit conditions before and after shaking. A comprehensive description of the model and experimental conditions is given by Kutter et al. (2018).

A total of 24 centrifuge test replicas of the sloping deposit were conducted at the 9 centrifuge facilities during LEAP-2017 (Carey et al. 2019; Escoffier and Audrain 2019; Hung and Liao 2019; Kim et al. 2019; Korre et al. 2019; Liu et al. 2019; Madabhushi et al. 2019; Okamura and Nurani Sjafruddin 2019; Vargas Tapia et al. 2019). The centrifuge models were subjected to input motions aimed at achieving base accelerations with different levels of closeness to a prescribed reference motion, as shown in Fig. 7.3. This figure also shows the input motions of two tests termed RPI0 and RPI4. RPI0 was conducted during LEAP-2015 (Kokkali et al. 2019). RPI0 and RPI1 were intended to be replica of each other. RPI4 was conducted in 2018 and was planned to be for a loose soil model (with an achieved $D_{\mathrm{r}}=40 \%$ ). A qualitative assessment of the recorded motions reveals that the obtained input accelerations have different levels of similarities and differences. These differences are due to variability in equipment (e.g., shaker actuators) along with other unknown uncertainties and lead to dissimilarities in input amplitude, phase, and frequency contents. The recorded soil accelerations also showed a significant level of variability among the different centrifuge tests, as illustrated by the AH4 motions in Fig. 7.4. In addition to the dispersion in input motions, the response accelerations were also affected by the variability in properties and characteristics of the analyzed soil deposits (such as the relative density, as shown in Fig. 7.2), which, from a broad perspective, may lead to amplification, de-amplification, changes in response frequency and phase, and possibly other aspects of variations in soil accelerations.

\subsection{Difference Metrics}

A validation exercise of soil liquefaction computational tools involves (in addition to other requisites (Oberkampf and Roy 2010)) (1) an assessment of the similarities and differences in achieved input motions and recorded responses and (2) a sensitivity analysis to quantify how the different uncertainties in input and initial conditions affect soil response. Metrics are needed to quantify the level of consistency in input motions, compare the responses of the test replicas, and evaluate the level of agreement between experimental and numerical results. A number of metrics 


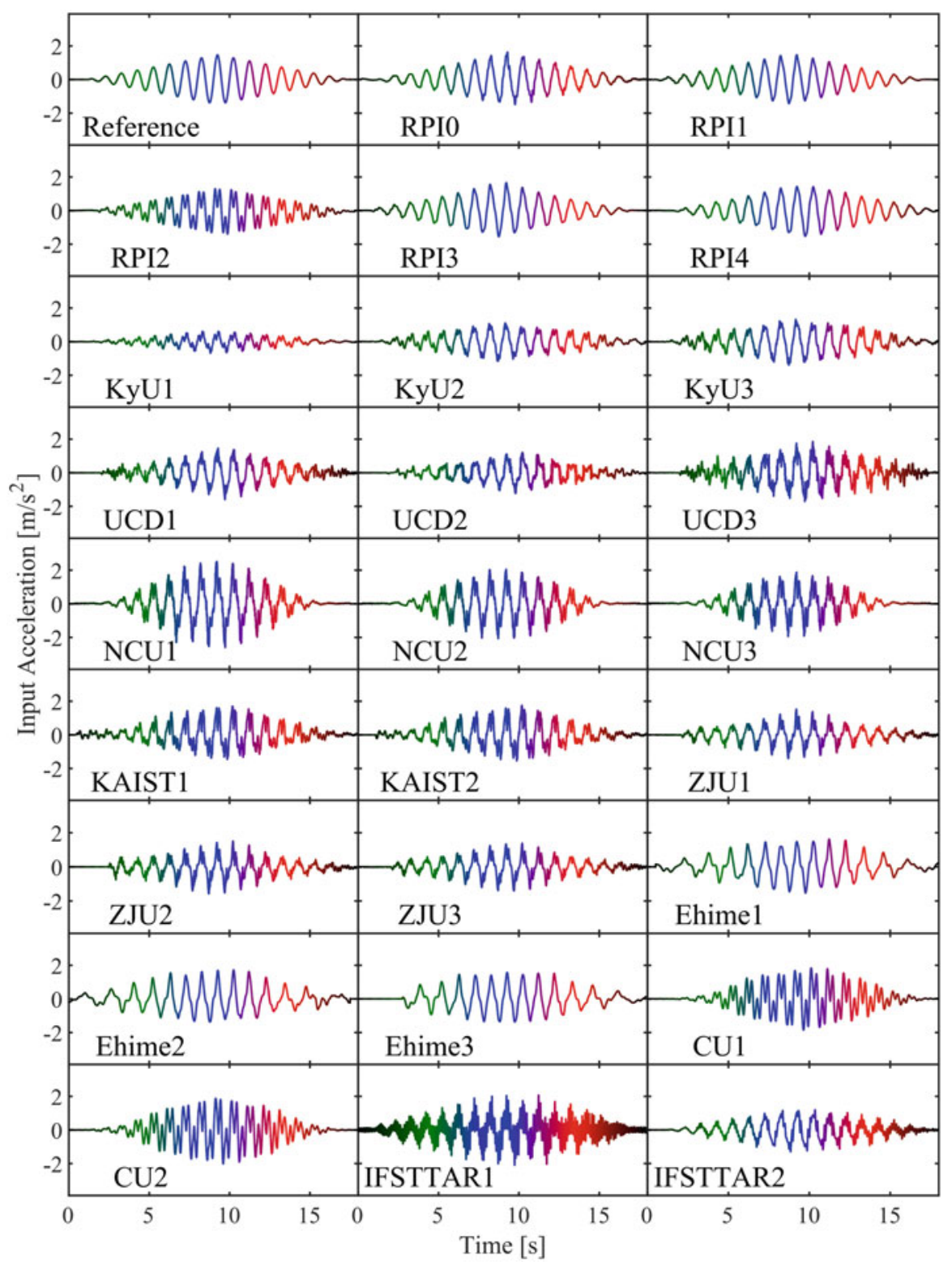

Fig. 7.3 Reference and achieved input (AH11) accelerations of the analyzed 26 centrifuge tests

have been used by researchers to assess differences among dynamic time histories (e.g., accelerations), ranging from a simple vector norm to the Sprague and Geers metric (Geers 1984) which identifies magnitude and phase difference components, along with others. A brief overview of these metrics and some of the associated 


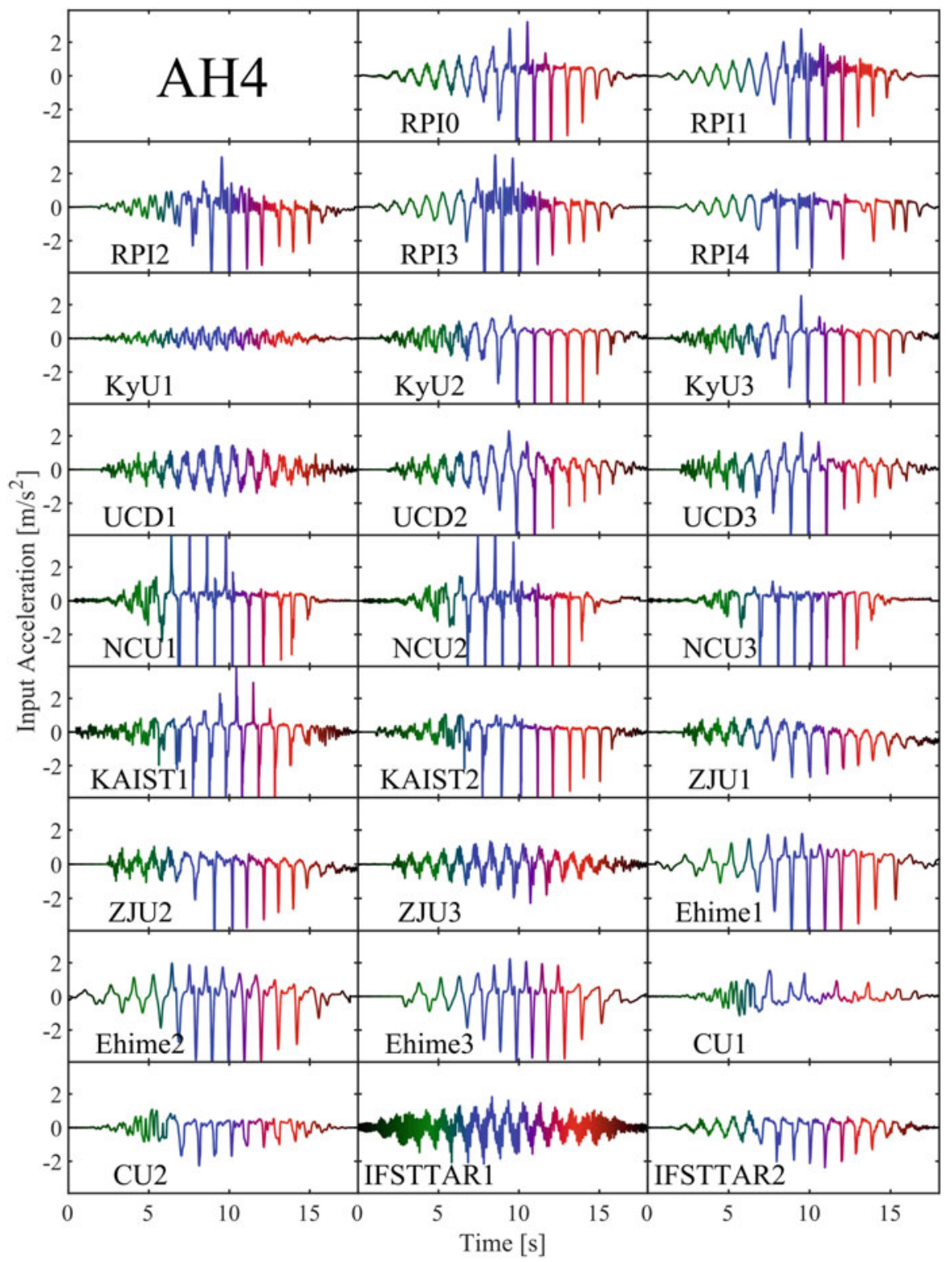

Fig. 7.4 Recorded AH4 soil accelerations of the analyzed 26 centrifuge tests

characteristics are discussed by Zeghal et al. (2018). This article uses a new approach (Zeghal et al. 2018). The difference $d_{i j}$ between two corresponding acceleration time histories $a_{i}=a_{i}(t)$ and $a_{j}=a_{j}(t)$ of two different test replicas $i$ and $j$ is quantified using a normalized mean squared deviation (MSD): 


$$
d_{i j}=\frac{\int_{0}^{W}\left(a_{i}-a_{j}\right)^{2} d t}{2\left(\int_{0}^{W} a_{i}^{2} d t+\int_{0}^{W} a_{j}^{2} d t\right)}
$$

in which $t$ is time and $W$ is length of a time window of interest. This metric is normalized so that it varies between 0 and 1 . A $d_{i j}$ metric approaching zero means that the two accelerations are essentially the same, whereas a metric of 1 is obtained, for instance, when two pure sinusoidal motions are 180 degrees out of phase with each other. The measure $d_{i j}$ is decomposed in terms of four specific fundamental components, namely, phase, frequency shift, amplitude at $1 \mathrm{~Hz}$, and amplitude of frequency components higher than $2 \mathrm{~Hz}$ (referred to as $2+\mathrm{Hz}$ ):

$$
d_{i j}=d_{i j}^{\text {phase }}+d_{i j}^{\text {shape }}+d_{i j}^{F \text { shift }}
$$

with:

$$
\begin{gathered}
d_{i j}^{\text {phase }}=\frac{\int_{0}^{+\infty}\left[2\left|A_{i}\right|\left|A_{j}\right|-\left(A_{i} A_{j}^{*}+A_{i}^{*} A_{j}\right)\right] d f}{2\left(\int_{0}^{+\infty} A_{i}^{2} d f+\int_{0}^{+\infty} A_{j}^{2} d f\right)} \\
d_{i j}^{\text {shape }}=\frac{D F W\left(\left|A_{i}\right|,\left|A_{j}\right|\right)}{2\left(\int_{0}^{+\infty} A_{i}^{2} d f+\int_{0}^{+\infty} A_{j}^{2} d f\right)} \\
d_{i j}^{F \text { shift }}=\frac{\int_{0}^{+\infty}\left(\left|A_{i}\right|-\left|A_{j}\right|\right)^{2} d f}{2\left(\int_{0}^{+\infty} A_{i}^{2} d f+\int_{0}^{+\infty} A_{j}^{2} d f\right)}-d_{i j}^{\text {shape }}
\end{gathered}
$$

in which $f$ is frequency; $A_{i}$ and $A_{j}$ are the Fourier transforms of $a_{i}$ and $a_{j}$, respectively; and $A_{i}^{*}$ refers to the complex conjugate of $A_{i}$. The phase component $d_{i j}^{\text {phase }}$ reflects differences due to dissimilarities in acceleration phase angles. The shape component $d_{i j}^{\text {shape }}$ quantifies the difference associated with the geometrical shape (i.e., wave form and amplitude). DFW refers to a dynamic frequency warping (Goswami 2019), which is similar in concept to the dynamic time warping (DTW) used in speech recognition (Rabiner and Huang 1993). The use of DFW enables isolation of the magnitude differences associated with (slight) shifts in acceleration frequencies. For the LEAP-2017 input accelerations, the shape components were decomposed further: 


$$
d_{i j}^{\text {shape }}=d_{i j}^{\text {shape }(1 \mathrm{~Hz})}+d_{i j}^{\text {shape }(2+\mathrm{Hz})}
$$

in which $d_{i j}^{\text {shape }(1 \mathrm{~Hz})}$ quantifies the shape (i.e., amplitude in this case) differences for the dominant $1 \mathrm{~Hz}$ component and $d_{i j}^{\text {shape }(2+\mathrm{Hz})}$ quantifies the difference related to the components at frequencies higher than $2 \mathrm{~Hz}$ (with the largest contribution often associated with the $3 \mathrm{~Hz}$ component). The frequency shift component $d_{i j}^{F \text { shift }}$ evaluates the difference stemming from variability in frequency of the acceleration components.

These metrics were verified using simple synthetic acceleration time histories with prescribed differences and were found to be effective (difference) identification and quantification tools (Goswami 2019). Also, the four difference metrics were used to evaluate equivalent (average) differences in amplitude at $1 \mathrm{~Hz}$, amplitude for the $2+\mathrm{Hz}$ components, phase angle, and a shift in frequency at $1 \mathrm{~Hz}$ (referred to as $\Delta A_{i j}^{1 \mathrm{~Hz}}, \Delta A_{i j}^{2+\mathrm{Hz}}, \Delta \Phi_{i j}$, and $\Delta F_{i j}$, respectively) to characterize and quantify the specific factors responsible for the observed differences in accelerations. The details of this evaluation are presented in Goswami (2019). The relative values of the different metrics $d_{i j}^{\text {shape }(1 \mathrm{~Hz})}, d_{i j}^{\text {shape }(2+\mathrm{Hz})}, d_{i j}^{\text {phase }}$, and $d_{i j}^{F \text { shift }}$ and the differences $\Delta A_{i j}^{1 \mathrm{~Hz}}, \Delta A_{i j}^{2+\mathrm{Hz}}$, $\Delta \Phi_{i j}$, and $\Delta F_{i j}$ can be used as indicators to ascertain the difference that prevails.

\subsubsection{Input Motion Differences}

An analysis was conducted to assess the differences among the reference acceleration and the input motions that were recorded during the 26 centrifuge tests. First, the input motions were all cross-correlated to determine a consistent common time $t=0$ for all the experiments. This eliminated all phase differences that are associated with a simple shift in the origin of time. The computed total differences $d_{i j}$ provided quantitative measures with numerical values varying from about 0.01 to 0.25, as exhibited in Fig. 7.5. In this figure, a 3D bar graph is used to display the whole set of difference metrics among the 26 recorded accelerations and the reference motion.

Overall, the input accelerations can be divided into three groups according to the difference metric values (Fig. 7.5): (1) Group 1 of accelerations that closely match the reference motion and also each other with $d_{i j}=0.00$ to about 0.03 , (2) Group 2 of accelerations that have an average match to the reference motion (and among the group) with $d_{i j}=$ about 0.03 to 0.08 , and (3) Group 3 of accelerations that do not closely match the reference motion and also each other with $d_{i j}=$ about 0.08 to 0.25 . Note that Group 3 includes the motion RPI3 (corresponding to the acceleration termed 5 in Fig. 7.5) which was generated so that it includes intentionally a significant $3 \mathrm{~Hz}$ component. The motions that are in a close match provide information that may be used, for instance, to assess reproducibility, while those with a loose match are advantageous in evaluating the sensitivities of the experiments to variation in input motions. 

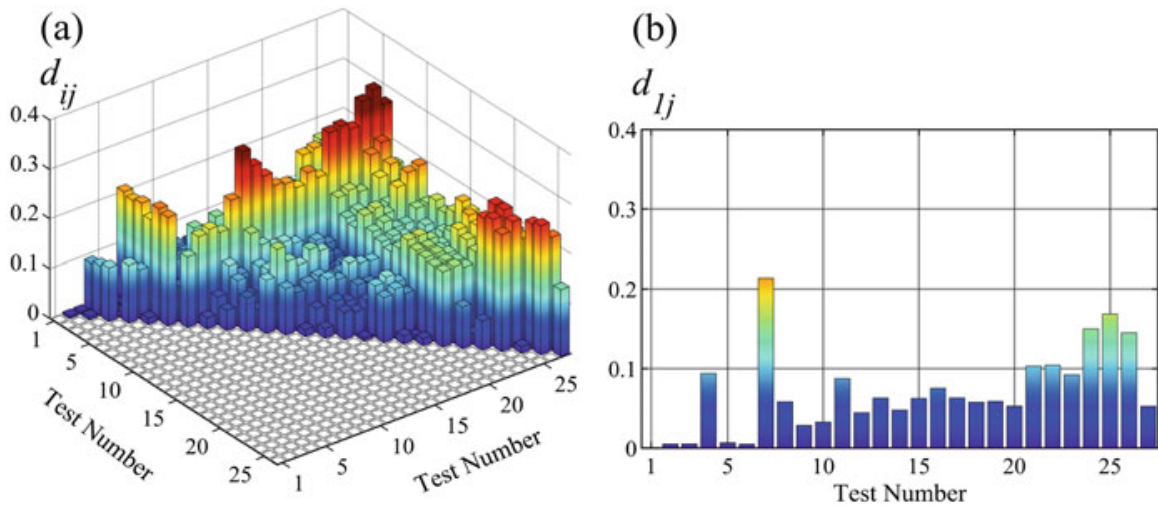

Fig. 7.5 Input motion difference metrics: (a) among the reference motion (termed 1) and all analyzed centrifuge input accelerations (termed 2 to 26) and (b) between the reference motion and recorded accelerations (corresponding to the first (back) row of the left figure)

The total difference metrics were decomposed into $1 \mathrm{~Hz}$ amplitude, $2+\mathrm{Hz}$ amplitude, phase, and frequency shift component measures to assess the nature of the associated dissimilarities and reasons for these differences, as shown in Fig. 7.6. This figure also shows the quantitative values of the corresponding differences $\Delta A_{i j}^{1 \mathrm{~Hz}}, \Delta A_{i j}^{2+\mathrm{Hz}}, \Delta \Phi_{i j}$, and $\Delta F_{i j}$ among the accelerations. The decomposition shows that the difference metrics are overall comparable in values, with $d_{i j}^{\text {shape }(1 \mathrm{~Hz})}$ being somewhat lower and $d_{i j}^{F \text { shift }}$ slightly higher than the other metrics. There is, however, a group of about five input accelerations that have relatively larger difference metrics (than the rest of the motions). The $d_{i j}^{\text {shape }(2+\mathrm{Hz})}$ values clearly show that there are four input motions which have larger difference metrics with the rest of the accelerations and are consistent with the visual assessment provided by Fig. 7.3. Figure 7.6 also shows the quantitative values of the differences $\Delta A_{i j}^{1 \mathrm{~Hz}}, \Delta A_{i j}^{2+\mathrm{Hz}}, \Delta \Phi_{i j}$, and $\Delta F_{i j}$ among the accelerations. The differences in phase angle $\Delta \Phi_{i j}$ and frequency $\Delta F_{i j}$ are relatively minor from a practical perspective. The substantially low values of $\Delta \Phi_{i j}$ are explained by the use (in the conducted analyses) of a consistent common time $t=0$ for all the experiments and the fact that all experiments properly achieved an input motion with a dominant $1 \mathrm{~Hz}$ component. The $\Delta A_{i j}^{1 \mathrm{~Hz}}$ and $\Delta A_{i j}^{2+\mathrm{Hz}}$ were more significant (especially for a set of about six input motions), even though the corresponding difference metrics were not substantially large. This is explained by the lower sensitivity of the total difference metric $d_{i j}$ to $\Delta A_{i j}^{1 \mathrm{~Hz}}$ and $\Delta A_{i j}^{2+\mathrm{Hz}}$ (compared to $\Delta \Phi_{i j}$ and $\Delta F_{i j}$ ). Overall, the $\Delta A_{i j}^{2+\mathrm{Hz}}$ values were larger than those of $\Delta A_{i j}^{1 \mathrm{~Hz}}$ and were as large as $0.6 \mathrm{~m} / \mathrm{s}^{2}$. A summary of the differences $\Delta A_{i j}^{1 \mathrm{~Hz}}$ and $\Delta A_{i j}^{2+\mathrm{Hz}}$ between the reference and input motions is presented in Fig. 7.7. 

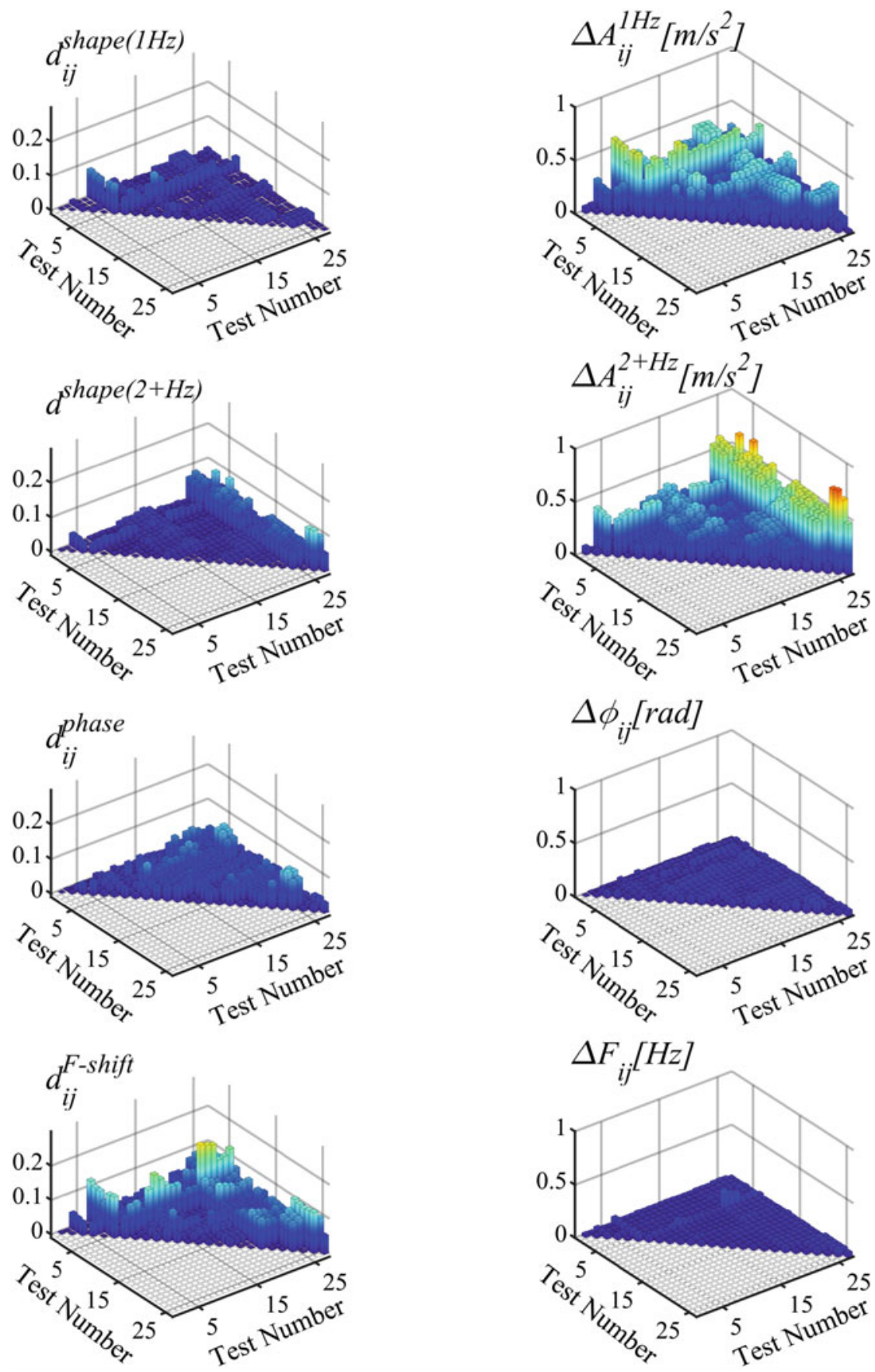

Fig. 7.6 Difference metric components of the reference and input accelerations and corresponding $\Delta A_{i j}^{1 \mathrm{~Hz}}, \Delta A_{i j}^{2+\mathrm{Hz}}, \Delta \Phi_{i j}$, and $\Delta F_{i j}$ 

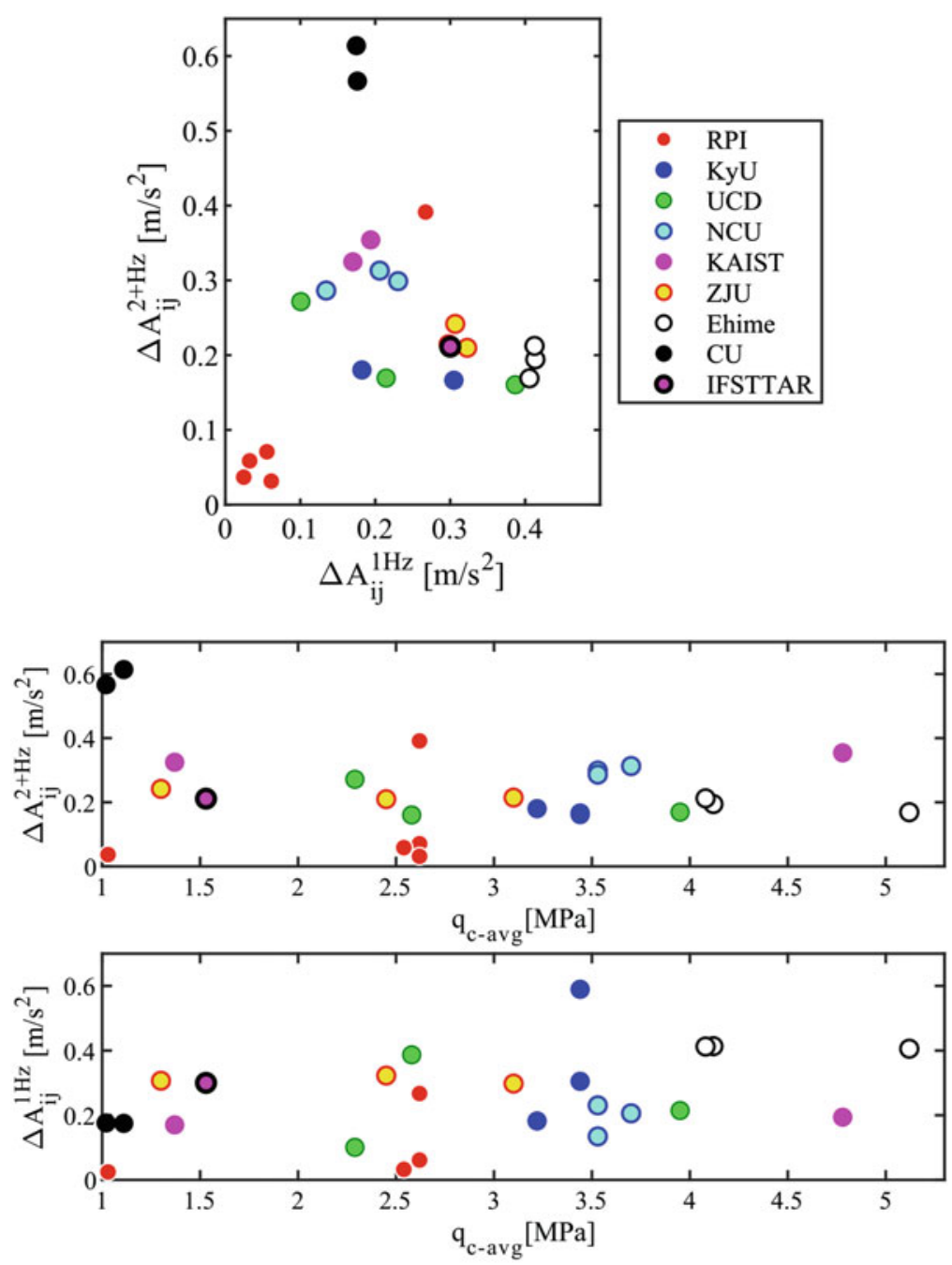

Fig. 7.7 Summary of differences in input motion and initial conditions among the 26 replica tests (in terms $\Delta A_{i j}^{1 \mathrm{~Hz}}$ and $\Delta A_{i j}^{2+\mathrm{Hz}}$ of the base motions and cone penetration resistance $q_{\mathrm{c} \text {-avg }}$ )

\subsubsection{Response Motion Differences}

The total discrepancy measures and corresponding components were evaluated for the recorded soil responses at different depths. Herein, only the total metrics are presented and discussed (because of space limitations). The decomposition of these metrics and additional details are given in Goswami (2019). The values of the difference metric for the accelerations AH1 to AH4 (Fig. 7.8) increased from the base of the deposit to the free surface and were considerable at the shallow depth location AH4 (reaching values 
(a)
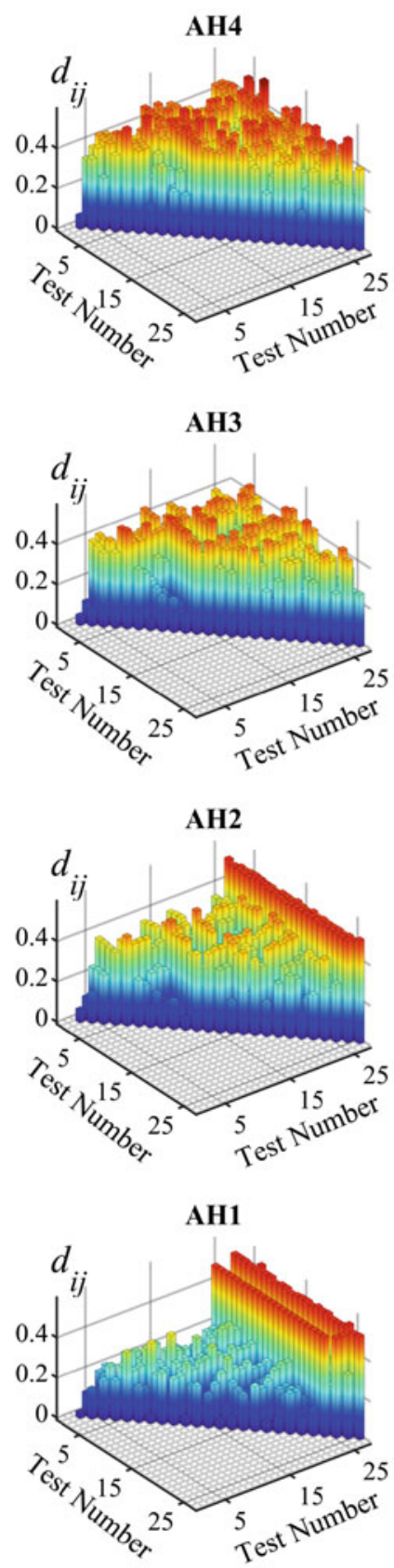

(b)
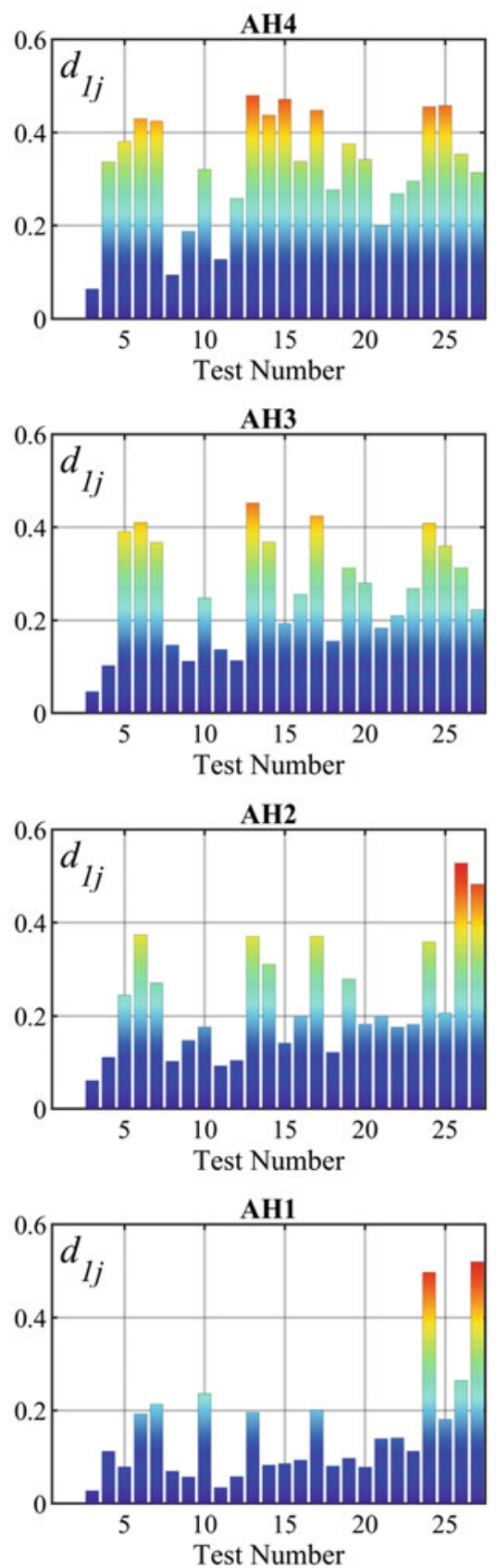

Fig. 7.8 Response motion difference metrics for AH1, AH2, AH3, and AH4: (a) among the analyzed 26 centrifuge test accelerations and (b) between RPI0 and the other tests 
of about 0.5 ). The difference metrics between the RPI0 accelerations and the other tests are shown in Fig. 7.8b to illustrate the range and level of likeliness and dissimilarities that these tests had with the response of one of the tests with an input motion close to the reference. These dissimilarities provide valuable information and are used below to assess the sensitivity and relative effects of the initial conditions on the difference metrics for the response acceleration at different depths.

\subsection{Sensitivity Analysis}

Evaluation of reliable estimates of the response (e.g., acceleration, displacement, etc.) sensitivity of a physical system, such as the analyzed LEAP sloping deposit, using a relatively limited set of test data represents a significant challenge. Diverse approaches have been used in a number of fields to evaluate local and global system sensitivities. A local sensitivity is evaluated based on a numerical differentiation concept and is usually obtained as the simple ratio of (observed or evaluated) changes in response quantities to a corresponding small variation in input parameters (at a specific value of these parameters). In contrast, a global sensitivity is based on a statistical framework and considers a range of input variations (in contrast to small variations). A number of review papers have been dedicated to these topics (e.g., Iooss and Lemaitre 2015). In this study, the local approach is not adequate in view of the involved experimental uncertainties and limitations, while the common global approaches are hindered by the limited amount (from a statistical point of view) of experimental data available. The sensitivities were therefore estimated on the basis of a kriging analysis.

Kriging is a semi-parametric Gaussian process regression method that was originally developed in the field of geostatistics (Chilès and Delfiner 1999; McCullough et al. 2017). It provides an effective means that may be employed to estimate response quantities, and corresponding derivatives and integrals, over a domain of associated input parameters using only noisy observations or measurements for a limited irregularly spaced set of these parameters. The method involves an averaging process and provides an estimate of uncertainty (in terms of a standard deviation). Herein, kriging was used to assess the sensitivity of the difference metrics of the recorded $\mathrm{AH} 1$ to $\mathrm{AH} 4$ accelerations to variations in input motion and initial soil fabric condition associated with soil deposition and achieved grain packing.

\subsubsection{Acceleration Sensitivity}

The difference analysis above showed that $\Delta A_{i j}^{1 \mathrm{~Hz}}$ and $\Delta A_{i j}^{2+\mathrm{Hz}}$ are the two main input motion parameters that varied during the analyzed 26 LEAP centrifuge tests. The tested soil models also had variability in soil deposition and achieved grain packing (as documented by the mass densities reported by the different centrifuge 
facilities (Kutter et al. 2018, 2019)). These variations have a direct effect, for instance, on stiffness properties which in turn affect the deposit response. An average over depth of the CPT (cone penetration test) tip resistance is deemed herein to correlate better with the deposit initial fabric and packing conditions than relative or mass densities (mainly due to the high sensitivity of density computation to errors in the measurement of volume). The average of the measured CPT resistance at $1.5 \mathrm{~m}$, $2.0 \mathrm{~m}, 2.5 \mathrm{~m}$, and $3.0 \mathrm{~m}$ depths (hereafter referred to as $q_{\text {c-avg }}$ ) for the different centrifuge tests is presented in Fig. 7.7 along with the differences in input motion.

A kriging analysis was conducted to assess the sensitivity of the recorded accelerations to the parameters $\Delta A_{i j}^{1 \mathrm{~Hz}}, \Delta A_{i j}^{2+\mathrm{Hz}}$, and $q_{\mathrm{c} \text {-avg. }}$. The sensitivity analysis was performed using a subset of 17 tests of the conducted 26 centrifuge experiments. The tests were selected based on an investigation of the associated stress and strain time histories. This investigation showed a consistency among the stress-strain response of these tests and fundamental differences with the remaining nine tests, as shown in Fig. 7.9. The details of the stress-strain analysis, rational for the 17 test selection, and details of the kriging analysis are given in Goswami (2019). The following paragraphs focus on the conducted analysis results for brevity.

The sensitivity analysis is based on an estimate of the variation of the total difference measures $d_{i j}$ of the recorded accelerations at $\mathrm{AH} 1$ to $\mathrm{AH} 4$ as a function of $\Delta A_{i j}^{1 \mathrm{~Hz}}, \Delta A_{i j}^{2+\mathrm{Hz}}$, and $q_{\mathrm{c} \text {-avg. }}$. Specifically, the analysis provided a kriging hypersurface representing $d_{i j}$ (for each of $\mathrm{AH} 1$ to $\mathrm{AH} 4$ ) as a function of the variables $\Delta A_{i j}^{1 \mathrm{~Hz}}, \Delta A_{i j}^{2+\mathrm{Hz}}$, and $q_{\mathrm{c}-\text { avg }}$ (over the domain associated with these variables, as shown in Fig. 7.7). The differences among the tests in input motions, $\Delta A_{i j}^{1 \mathrm{~Hz}}$ and $\Delta A_{i j}^{2+\mathrm{Hz}}$, and in response metric (at $\mathrm{AH} 1$ to $\mathrm{AH} 4$ ), $d_{i j}$, had to be computed with respect to a common reference, which was selected to be the RPI1 test. This test had an input acceleration substantially close to the reference and a relative density close to the reference mean value (Fig. 7.2). Other tests could also be used as a reference and would lead similar results to the ones presented below.

Three sets of surfaces and corresponding sensitivity functions are employed herein to visualize the obtained $d_{i j}$ kriging hypersurface results, as shown in Figs. 7.10, 7.11, 7.12, 7.13, 7.14, and 7.15. Thus, Fig. 7.10 shows the difference metric variations as a function of $\Delta A_{i j}^{1 \mathrm{~Hz}}$ and $\Delta A_{i j}^{2+\mathrm{Hz}}$ for a $q_{\mathrm{c} \text { avg }}=2.6 \mathrm{MPa}$. This value corresponds to the mid-point of the domain of variations for $q_{\mathrm{c} \text {-avg }}$ and corresponds to a $D_{\mathrm{r}}$ of about $65 \%$. The obtained results show that the AH1 accelerations have comparable sensitivities to variations in $\Delta A_{i j}^{1 \mathrm{~Hz}}$ and $\Delta A_{i j}^{2+\mathrm{Hz}}$ (for $q_{\mathrm{c} \text {-avg }}=2.7 \mathrm{MPa}$ ) and the associated discrepancy metric practically varies linearly as a function of these two parameters, as shown in Fig. 7.10. In contrast, the response at $\mathrm{AH} 4$ is about two times as sensitive to a $\Delta A_{i j}^{2+\mathrm{Hz}}$ as to $\Delta A_{i j}^{1 \mathrm{~Hz}}$. The discrepancy metrics for $\mathrm{AH} 1$ to $\mathrm{AH} 4$ show a sensitivity that increased from the bottom of the deposit to the free surface. The associated sensitivity functions $\partial d_{i j} / \partial A_{i j}^{1 \mathrm{~Hz}}$ and $\partial d_{i j} / \partial A_{i j}^{2+\mathrm{Hz}}$ for $q_{\mathrm{c}-\mathrm{avg}}=2.7 \mathrm{MPa}$ (Fig. 7.11) confirmed that AH1 has mostly constant sensitivity functions and $\mathrm{AH} 4$ has a sensitivity $\partial d_{i j} / \partial A_{i j}^{2+\mathrm{Hz}}$ with large variations (especially as a function of $\Delta A_{i j}^{2+\mathrm{Hz}}$ ). The sensitivities for $\mathrm{AH} 2$ and $\mathrm{AH} 3$ 


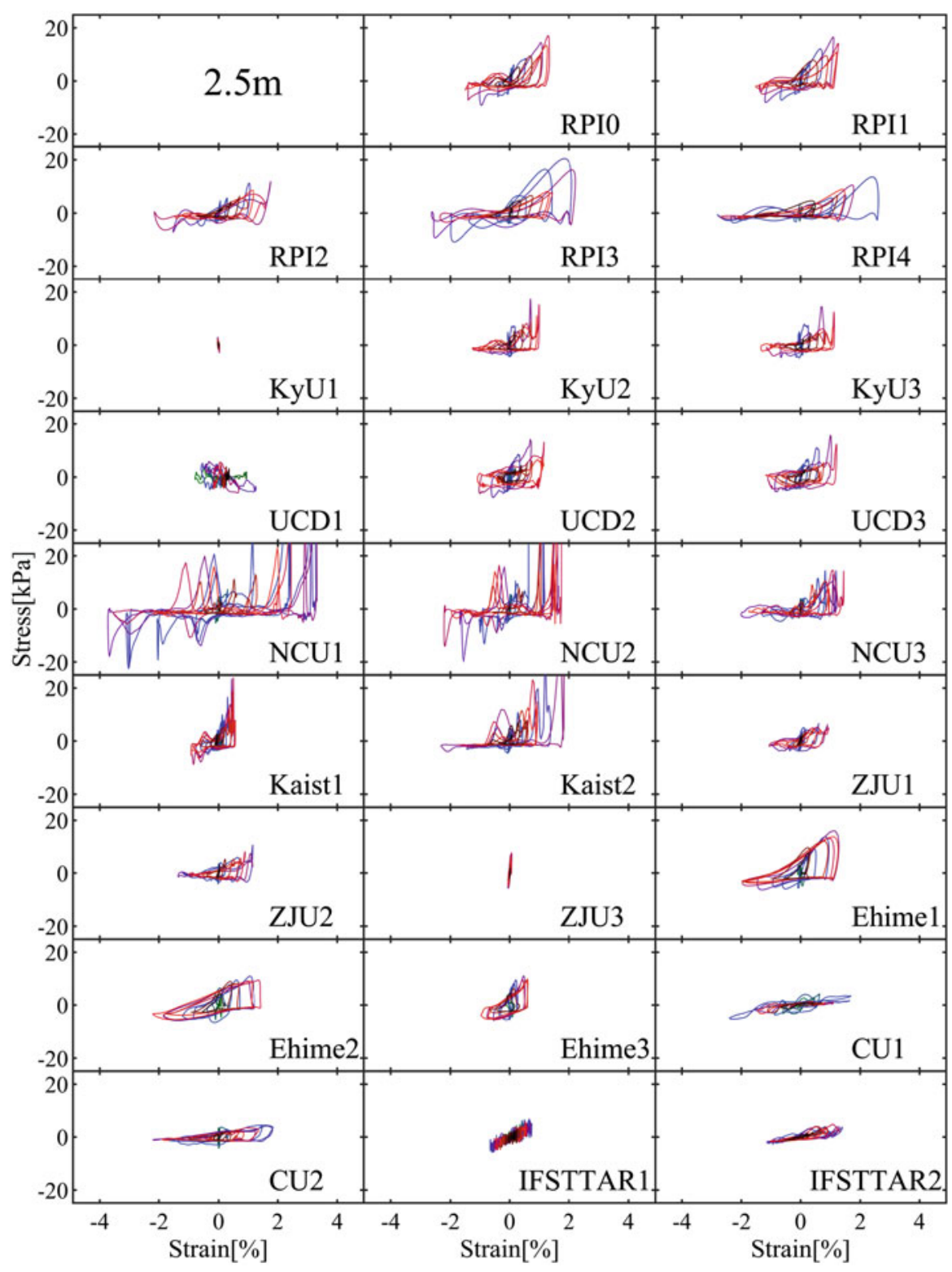

Fig. 7.9 Stress-strain response of the analyzed centrifuge tests (at $2.5 \mathrm{~m}$ depth along the central accelerometer array)

had values that varied between those of AH1 and AH4. Note however that the estimated variations of the discrepancy metric (and corresponding sensitivities) are associated with larger standard deviation (i.e., lower level of confidence) at large $\Delta A_{i j}^{2+\mathrm{Hz}}$ values, especially for $\mathrm{AH} 3$ and $\mathrm{AH} 4$. This is explained by the sparsity of 

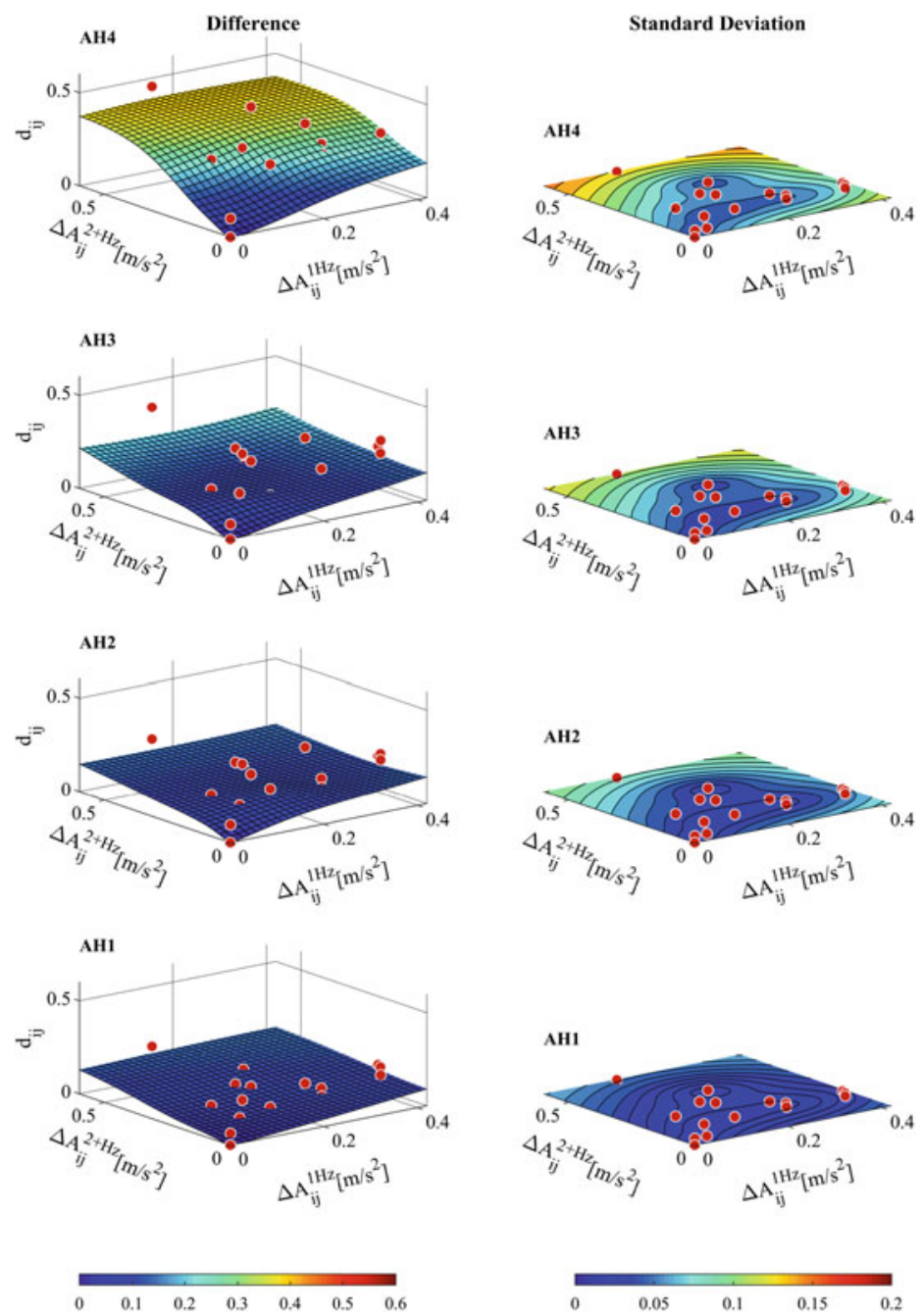

Fig. 7.10 Variation of the difference metric $d_{i j}$ of the recorded accelerations (at AH1 to AH4) as a function of $\Delta A_{i j}^{1 \mathrm{~Hz}}$ and $\Delta A_{i j}^{2+\mathrm{Hz}}$ for a $q_{\mathrm{c} \text {-avg }}=2.7 \mathrm{MPa}$ (the red dots correspond to the analyzed 17 tests)

data for large values of $\Delta A_{i j}^{2+\mathrm{Hz}}$ (only one experiment had a $\Delta A_{i j}^{2+\mathrm{Hz}}$ larger than $0.04 \mathrm{~m} / \mathrm{s}^{2}$ ).

The difference metric surfaces as a function of $\Delta A_{i j}^{1 \mathrm{~Hz}}$ and $q_{\mathrm{c}-\text { avg }}$ for $\Delta A_{i j}^{2+\mathrm{Hz}}=0$, and $\Delta A_{i j}^{2+\mathrm{Hz}}$ and $q_{\mathrm{c}-\text { avg }}$ for $\Delta A_{i j}^{1 \mathrm{~Hz}}=0$, and the corresponding sensitivity functions (Figs. 7.12, 7.13, 7.14, and 7.15) were employed to explore the effects of the observed variation in CPT resistance. The obtained metric surfaces and sensitivity 

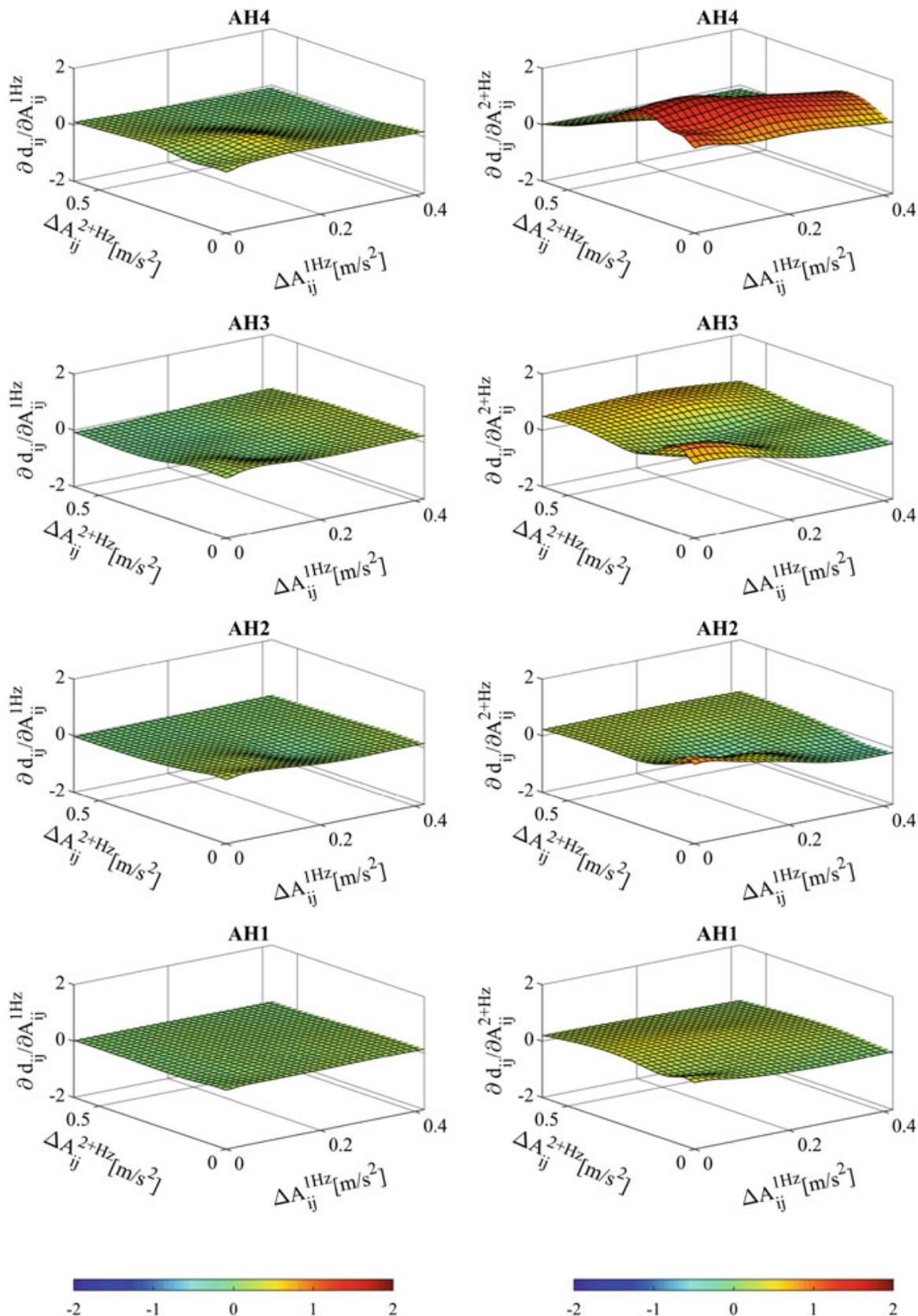

Fig. 7.11 Sensitivity functions of the total difference metric of the recorded accelerations (at AH1 to AH4) with respect to variations in $\Delta A_{i j}^{1 \mathrm{~Hz}}$ and $\Delta A_{i j}^{2+\mathrm{Hz}}$ for $q_{\mathrm{c} \text {-avg }}=2.7 \mathrm{MPa}$ 

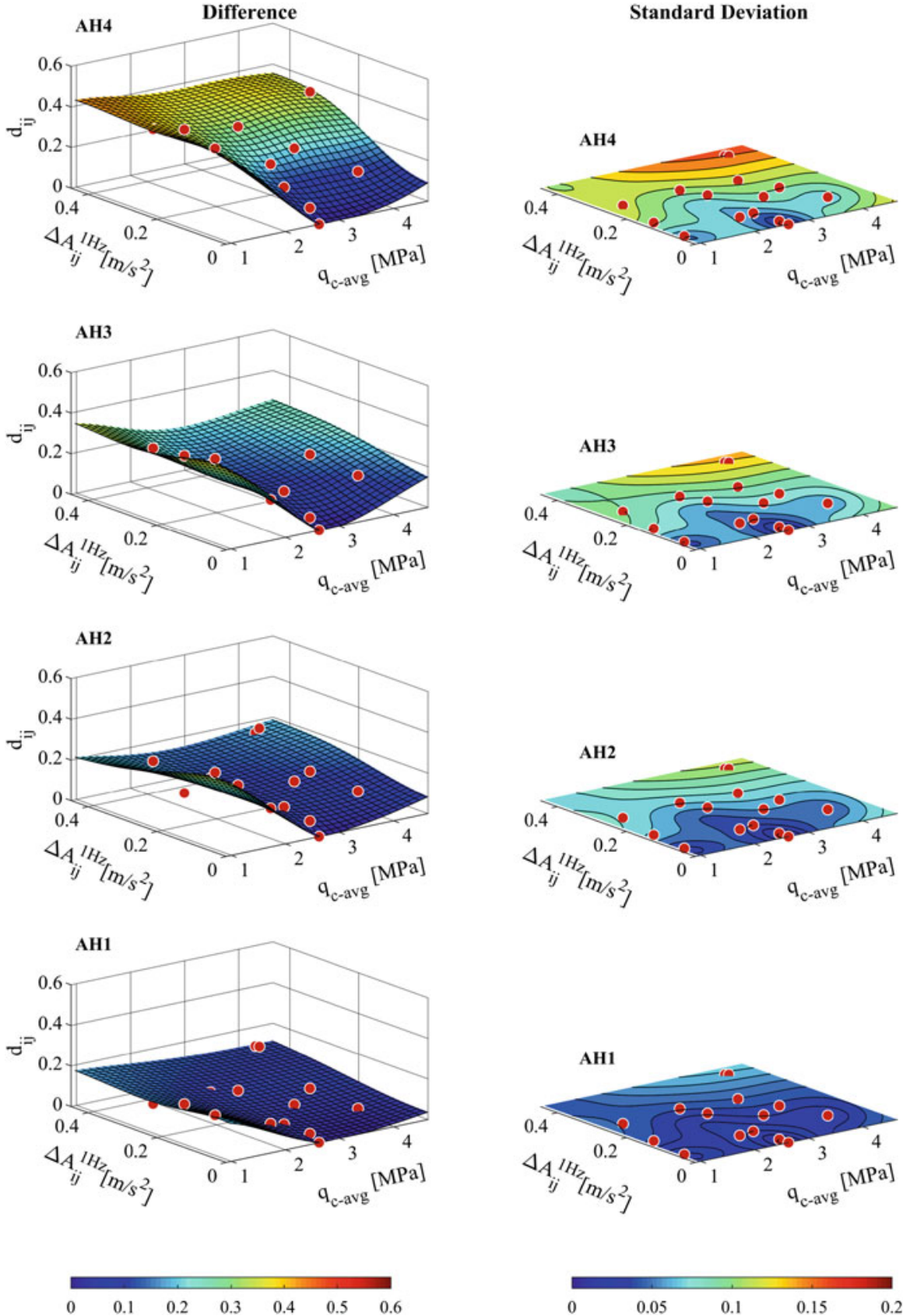

Fig. 7.12 Variation of the total difference metric of the recorded accelerations (at AH1 to AH4) as a function of $\Delta A_{i j}^{1 \mathrm{~Hz}}$ and $q_{\mathrm{c} \text {-avg }}$ for $\Delta A_{i j}^{2+\mathrm{Hz}}=0$ (the red dots correspond to the analyzed 17 tests) 

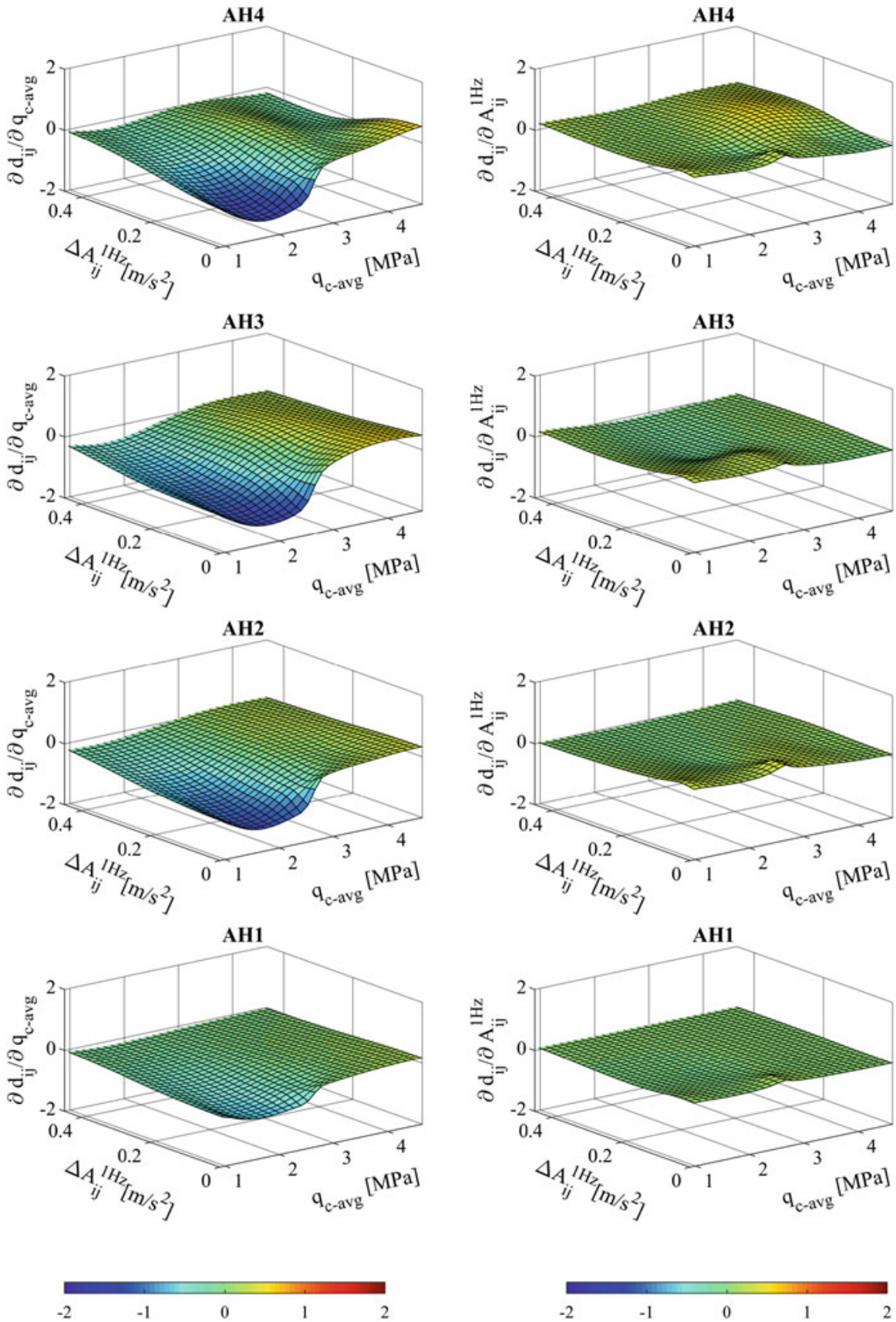

Fig. 7.13 Sensitivity functions of the total difference metric of the recorded accelerations (at AH1 to AH4) with respect to variations in $\Delta A_{i j}^{1 \mathrm{~Hz}}$ and $q_{\mathrm{c} \text {-avg }}$ for $\Delta A_{i j}^{2+\mathrm{Hz}}=0$ 

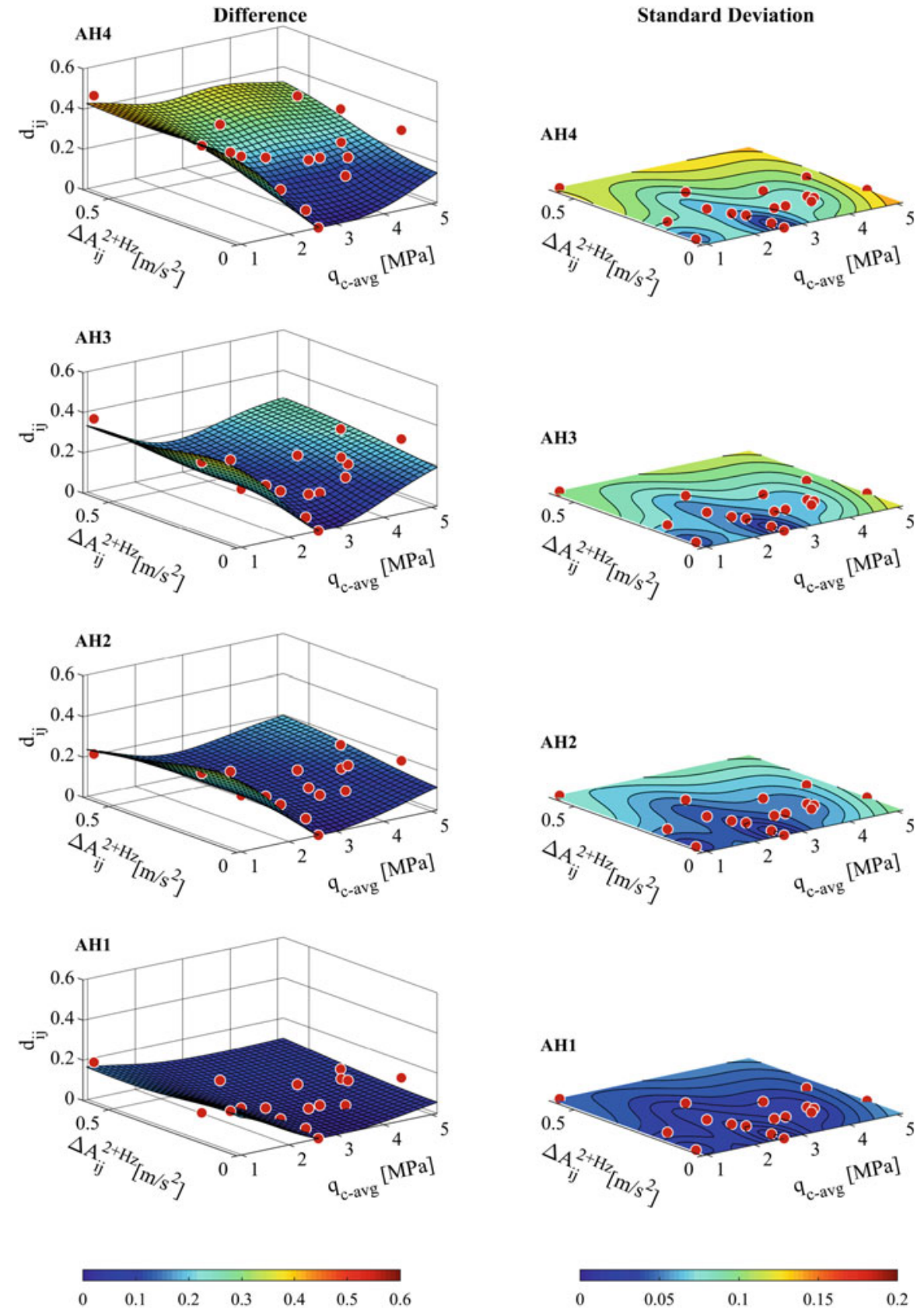

Fig. 7.14 Variation of the total difference metric of the recorded (AH1 to AH4) accelerations as a function of $\Delta A_{i j}^{2+\mathrm{Hz}}$ and $q_{\mathrm{c} \text {-avg }}$ for $\Delta A_{i j}^{1 \mathrm{~Hz}}=0$ and corresponding standard deviation (the red dots are the analyzed 17 tests) 



Fig. 7.15 Sensitivity functions of the total difference metric of the recorded accelerations (AH1 to AH4) with respect to variations in $\Delta A_{i j}^{2+\mathrm{Hz}}$ and $q_{\mathrm{c} \text {-avg }}$ for $\Delta A_{i j}^{1 \mathrm{~Hz}}=0$ 
functions show a response that is significantly more sensitive to a decrease in $q_{\mathrm{c} \text {-avg }}$ than an increase. This is explained by the fact that lower values of $q_{\mathrm{c} \text {-avg }}$ are associated with a looser more contractive soil with a response that contrasts substantially with that of the (dilative) reference deposit with a $D_{\mathrm{r}}$. of about $65 \%$. In contrast, larger $q_{\mathrm{c} \text {-avg }}$ values are indicative of a denser soil that is only slightly more dilative and has only a somewhat different response. The sensitivity values increased from $\mathrm{AH} 1$ to $\mathrm{AH} 4$, and the sensitivities with respect to $q_{\mathrm{c}-\text { avg }}$ were significantly larger than those associated with $\Delta A_{i j}^{1 \mathrm{~Hz}}$ and $\Delta A_{i j}^{2+\mathrm{Hz}}$. Overall, the obtained sensitivity functions (Figs. 7.11, 7.13, and 7.15) vary nonlinearly with variations in parameters. The level of nonlinearity increases from $\mathrm{AH} 1$ to $\mathrm{AH} 4$ and is more remarkable for the sensitivities that depends on $q_{\mathrm{c} \text {-avg. }}$. Figures $7.10,7.12$, and 7.14 also show the standard deviations corresponding to the estimated difference metric surfaces (and corresponding sensitivities). The deviations increased from AH1 near the bottom to AH4 close to the free surface of the deposit. Also, for any (acceleration) level, the deviations have the lowest values in the zones with well-distributed data points and largest values with sparse or no data points (as expected).

\subsubsection{Permanent Displacement Sensitivity}

A kriging analysis was used to assess the effects of variations in $\Delta A_{i j}^{1 \mathrm{~Hz}}, \Delta A_{i j}^{2+\mathrm{Hz}}$, and $q_{\mathrm{c}-\mathrm{avg}}$ on the permanent surface displacement (referred to as $D$ ). The analysis was performed for the selected 17 tests using the mean values of the measured displacement of the 2 central markers (Kutter et al. 2019). Three sets of surfaces are exhibited in Fig. 7.16 to visualize the obtained $D$ kriging hypersurface results as a function of (1) $\Delta A_{i j}^{1 \mathrm{~Hz}}$ and $\Delta A_{i j}^{2+\mathrm{Hz}}$ for a $q_{\mathrm{c} \text { avg }}=2.6 \mathrm{MPa}$, (2) $\Delta A_{i j}^{1 \mathrm{~Hz}}$ and $q_{\mathrm{c}-\text { avg }}$ for $\Delta A_{i j}^{2+\mathrm{Hz}}=0$, and (3) $\Delta A_{i j}^{2+\mathrm{Hz}}$ and $q_{\mathrm{c}-\text { avg }}$ for $\Delta A_{i j}^{1 \mathrm{~Hz}}=0$. The obtained results show that the measured permanent displacements are generally more sensitive to variations in $\Delta$ $A_{i j}^{1 \mathrm{~Hz}}$ and $\Delta A_{i j}^{2+\mathrm{Hz}}$ than $q_{\mathrm{c} \text {-avg. }}$. In fact the displacements have rather a low sensitivity to $q_{\mathrm{c}-\text { avg }}$ for $\Delta A_{i j}^{1 \mathrm{~Hz}} \approx 0$ and $\Delta A_{i j}^{2+\mathrm{Hz}} \approx 0$. The sensitivity to the CPT resistance increases when the variations in $q_{\mathrm{c} \text {-avg }}$ are combined with variations in $\Delta A_{i j}^{1 \mathrm{~Hz}}$ and $\Delta A_{i j}^{2+\mathrm{Hz}}$. The corresponding standard deviations (Fig. 7.16) were again reasonable in the zone with well-distributed data points, and high values were observed in areas with limited or no data points.

\subsection{Conclusions}

This article presented an analysis of the differences and sensitivities among the acceleration time histories of 26 centrifuge LEAP (Liquefaction Experiments and Analysis Projects) test replicas of a saturated sloping deposit. A normalized mean squared deviation is used as difference metric to quantify the dissimilarities between 


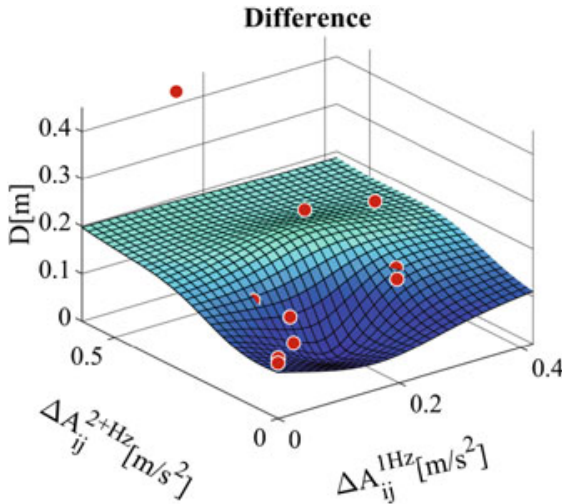

\section{Standard Deviation}
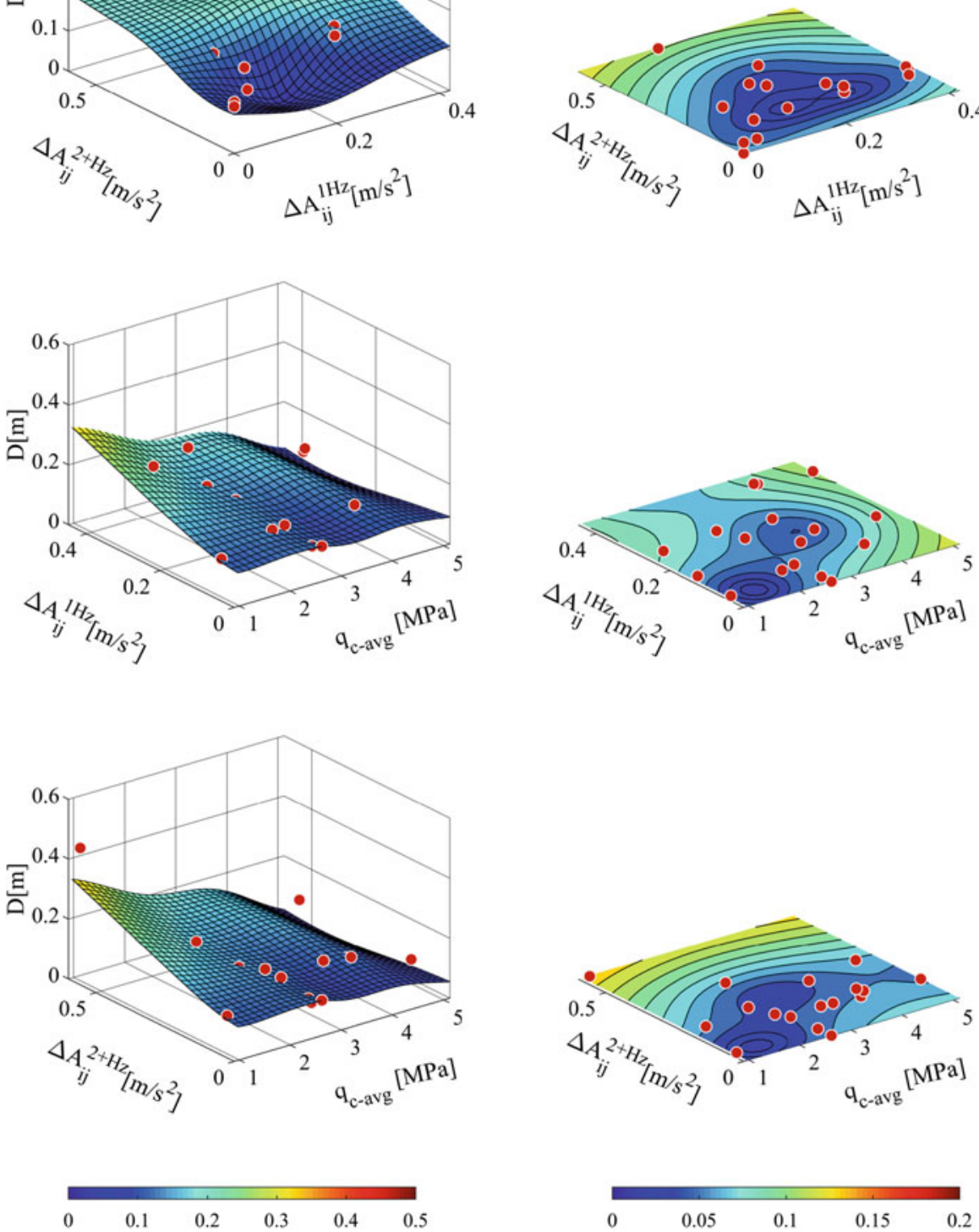

Fig. 7.16 Variation of the average (permanent) surface displacement $D$ as a function of $\Delta A_{i j}^{1 \mathrm{~Hz}}$ and $\Delta A_{i j}^{2+\mathrm{Hz}}$ for a $q_{\mathrm{c}}=2.7 \mathrm{MPa}, \Delta A_{i j}^{1 \mathrm{~Hz}}$ and $q_{\mathrm{c}-\text { avg }}$ for $\Delta A_{i j}^{2+\mathrm{Hz}}=0$, and $\Delta A_{i j}^{2+\mathrm{Hz}}$ and $q_{\mathrm{c}-\text { avg }}$ for $\Delta A_{i j}^{1 \mathrm{~Hz}}=0$ (the red dots are the analyzed 17 tests) 
recorded acceleration time histories. This metric is uniquely decomposed in four terms associated with phase, frequency shift, amplitude at $1 \mathrm{~Hz}$, and amplitude of frequencies higher than $2 \mathrm{~Hz}(2+\mathrm{Hz})$ components. These metrics and measures were employed to assess and quantify the discrepancies of the input and response accelerations of the 26 different test replicas. The obtained difference metric values showed that the input accelerations can be divided into three broad classes: (1) Group 1 of accelerations that closely match the reference and also each other, (2) Group 2 of accelerations that have an average match to the reference and among the group, and (3) Group 3 of accelerations that did not closely match the reference and also each other with. This broad range of motions provides valuable information to assess both the repeatability of the tests and sensitivity of the recorded responses to variations in input motion. The differences among input motions were found to be associated mostly with variation in amplitude of the dominant component at $1 \mathrm{~Hz}$ and the components with frequencies higher than $2 \mathrm{~Hz}(2+\mathrm{Hz})$.

A Gaussian process-based kriging was used to assess the sensitivity of the deposit response acceleration to differences in input motion amplitude at $1 \mathrm{~Hz}$ and $2+\mathrm{Hz}$ and average CPT (cone penetration test) resistance (used as a measure reflecting deposit fabric condition and initial grain packing). The conducted analyses showed that the analyzed deposit accelerations are relatively more sensitive to variations in CPT resistance than to the input motion and that this sensitivity is larger for a decrease in CPT resistance compared to an increase. The sensitivities were also found to be highly nonlinear functions of the variability in the input motion and CPT resistance. In contrast, the measured permanent displacements were generally more sensitive to differences in input motion amplitude at $1 \mathrm{~Hz}$ and $2+\mathrm{Hz}$ than the average CPT resistance.

Acknowledgments The experimental work of the LEAP project was supported by different institutions. In the USA, the work was funded by the US National Science Foundation Geotechnical Engineering program directed by Dr. Richard Fragaszy (NSF grants CMMI-1635524, CMMI1635307, and CMMI-1635040 to Rensselaer Polytechnic Institute, George Washington University, and University of California Davis, respectively). The work at Ehime U. was supported by JSPS KAKENHI Grant Number 17H00846. The work at Kyoto U. was supported by JSPS KAKENHI Grant Numbers 26282103, 5420502, and 17H00846. The work at Kansai U. was supported by JSPS KAKENHI Grant Number 17H00846. The work at KAIST was part of a project titled "Development of performance-based seismic design," funded by the Ministry of Oceans and Fisheries, Korea. The work at NCU was supported by MOST: 106-2628-E-008-004-MY3. The work at Zhejiang University was supported by the National Natural Science Foundation of China, Nos. 51578501 and 51778573; Zhejiang Provincial Natural Science Foundation of China, LR15E080001; and National Basic Research Program of China (973 Project), 2014CB047005. These supports are gratefully acknowledged.

\section{References}

Carey, T. J., Stone, N., Hajialilue Bonab, M., \& Kutter, B. L. (2019). LEAP-UCD-2017 centrifuge test at University of California, Davis. In B. Kutter et al. (Eds.), Model tests and numerical simulations of liquefaction and lateral spreading: LEAP-UCD-2017. New York: Springer. 
Chilès, J.-P., \& Delfiner, P. (1999). Geostatistics: Modeling spatial uncertainty. New York: Wiley. Escoffier, S., \& Audrain, P. (2019). LEAP-UCD-2017 centrifuge test at IFSTTAR. In B. Kutter et al. (Eds.), Model tests and numerical simulations of liquefaction and lateral spreading: LEAP-UCD-2017. New York: Springer.

Geers, T. (1984). Objective error measure for the comparison of calculated and measured transient response histories. Shock and Vibration Bulletin, 54, 99-102.

Goswami, N. (2019). Validation Framework for Assessment of numerical Predictions Using Leap Experiments. Troy, NY: PhD Thesis, Rensselaer Polytechnic Institute.

Hung, W.-Y., \& Liao, T.-W. (2019). LEAP-UCD-2017 centrifuge tests at NCU. In B. Kutter et al. (Eds.), Model tests and numerical simulations of liquefaction and lateral spreading: LEAP$U C D$-2017. New York: Springer.

Iooss, B., \& Lemaître, P. (2015). A review on global sensitivity analysis methods. In B. Iooss \& P. Lemaitre (Eds.), Uncertainty Management in Simulation-Optimization of Complex Systems (pp. 101-122). Boston, MA: Springer.

Kim, S.-N., Ha, J.-G., Lee, M.-G., \& Kim, D.-S. (2019). LEAP-UCD-2017 centrifuge test at KAIST. In B. Kutter et al. (Eds.), Model tests and numerical simulations of liquefaction and lateral spreading: LEAP-UCD-2017. New York: Springer.

Kokkali, P., Abdoun, T., \& Zeghal, M. (2019). Physical modeling of soil liquefaction: Overview of LEAP production test 1 at Rensselaer Polytechnic Institute. Soil Dynamics and Earthquake Engineering, 113, 629-649.

Korre, E., Abdoun, T., \& Zeghal, M. (2019). Verification of the repeatability of soil liquefaction centrifuge testing at Rensselaer. In B. Kutter et al. (Eds.), Model tests and numerical simulations of liquefaction and lateral spreading: LEAP-UCD-2017. New York: Springer.

Kutter, B. L., Carey, T. J., Zheng, B. L., Gavras, A., Stone, N., Zeghal, M., Abdoun, T., Korre, E., Manzari, M., Madabhushi, G. S., Haigh, S., Madabhushi, S. S., Okamura, M., Sjafuddin, A. N., Escoffier, S., Kim, D.-S., Kim, S.-N., Ha, J.-G., Tobita, T., Yatsugi, H., Ueda, K., Vargas, R. R., Hung, W.-Y., Liao, T.-W., Zhou, Y.-G., \& Liu, K. (2018). Twenty-Four Centrifuge Tests to Quantify Sensitivity of Lateral Spreading to Dr and PGA. In S. J. Brandenberg \& M. T. Manzari (Eds.), Geotechnical Earthquake Engineering and Soil Dynamics V, GSP 293 (pp. 383-393). Alexandria, VA: ASCE. https://doi.org/10.1061/9780784481486.040.

Kutter, B. L., Carey, T., Stone, N., Zheng, B. L., Gavras, A., Manzari, M. T., Zeghal, M., Abdoun, T., Korre, E., Escoffier, S., Haigh, S., Madabhushi, G., Madabhushi, S. S. C., Hung, W.-Y., Liao, T.-W., Kim, D.-S., Kim, S.-N., Ha, J.-G., Kim, N. R., Okamura, M., Sjafuddin, A. N., Tobita, T., Ueda, K., Vargas, R., Zhou, Y.-G., \& Liu, K. (2019). LEAP-UCD-2017 comparison of centrifuge test results. In B. Kutter et al. (Eds.), Model tests and numerical simulations of liquefaction and lateral spreading: LEAP-UCD-2017. New York: Springer.

Liu, K., Zhou, Y.-G., She, Y., Xia, P., Meng, D., Huang, J.-S., Yao, G., \& Chen, Y.-M. (2019). Specifications and results of centrifuge model test at Zhejiang University for LEAP-UCD-2017. In B. Kutter et al. (Eds.), Model tests and numerical simulations of liquefaction and lateral spreading: LEAP-UCD-2017. New York: Springer.

Madabhushi, S. S. C., Dobrisan, A., Beber, R., Haigh, S. K., \& Madabhushi, S. P. G. (2019). LEAPUCD-2017 centrifuge tests at Cambridge. In B. Kutter et al. (Eds.), Model tests and numerical simulations of liquefaction and lateral spreading: LEAP-UCD-2017. New York: Springer.

Manzari, M. T., El Ghoraiby, M. A., Kutter, B. L., Zeghal, M., Wang, R., Chen, R., Zhang, J.-M., Osamu Ozutsumi, O., Fukutake, K., Kiriyama, T., Fasano, G., Chiaradonna, A., Bilotta, E., Montgomery, J., Ziotopoulou, K., Chen, L., Ghofrani, A., Arduino, P., Wada, T., Ueda, K., Mercado, V., Fuentes, W., Lascarro, C., Yang, M., Barrero, A. R., Taiebat, M., Tsiaousi, D., Ugalde, J., Thaleia Travasarou, T., Ichii, K., Uemura, K., Orai, N., Hyodo, M., Abdoun, T., Haigh, S., Madabhushi, S., Tobia, T., Hung, W.-Y., Kim, D. S., Okamura, M., Zhou, Y.-G., \& Escoffier, S. (2018). Liquefaction experiment and analysis projects (LEAP): Summary of observations from the planning phase. Soil Dynamics and Earthquake Engineering, 113, 714-743. 
McCullough, M., Jayakumar, P., Dasch, J., \& Gorsich, D. (2017). The next generation NATO reference mobility model development. Journal of Terramechanics, 73, 49-60.

Oberkampf, W. L., \& Roy, C. J. (2010). Verification and validation in scientific computing. New York, NY: Cambridge University Press.

Okamura, M., \& Nurani Sjafruddin, A. (2019). LEAP-2017 centrifuge test at Ehime University. In B. Kutter et al. (Eds.), Model tests and numerical simulations of liquefaction and lateral spreading: LEAP-UCD-2017. New York: Springer.

Rabiner, L., \& Huang, B. (1993). Fundamentals of speech recognition. Eaglewood Cliffs, NJ: PTR Prentice Hall.

Vargas Tapia, R. R., Tobita, T., Ueda, K., \& Yatsugi, H. (2019). LEAP-UCD-2017 centrifuge test at Kyoto University. In B. Kutter et al. (Eds.), Model tests and numerical simulations of liquefaction and lateral spreading: LEAP-UCD-2017. New York: Springer.

Zeghal, M., Goswami, N., Manzari, M., \& Kutter, B. (2018). Discrepancy metrics and sensitivity analysis of dynamic soil response. Austin, TX: Geotechnical Earthquakes and Soil Dynamics.

Open Access This chapter is licensed under the terms of the Creative Commons Attribution 4.0 International License (http://creativecommons.org/licenses/by/4.0/), which permits use, sharing, adaptation, distribution and reproduction in any medium or format, as long as you give appropriate credit to the original author(s) and the source, provide a link to the Creative Commons license and indicate if changes were made.

The images or other third party material in this chapter are included in the chapter's Creative Commons license, unless indicated otherwise in a credit line to the material. If material is not included in the chapter's Creative Commons license and your intended use is not permitted by statutory regulation or exceeds the permitted use, you will need to obtain permission directly from the copyright holder. 